\title{
Ein Besuch in den Königlichen Gewächshäusern in Laeken (Brüssel)
}

\author{
Hilke Steinecke \& Willi WöBer
}

\begin{abstract}
The royal greenhouses of Laeken are built between 1874 and 1905. The large complex of glass houses was commissioned by King LEOPOLD II., son of LEOPOLD I., the first king of Belgium. The royal greenhouses are opened for public visitors every year from middle of april to the beginning of may. Palm house, winter garden and the other impressive glass houses host a large plant collection. Some of the palms, camellias and Citrus plants are due to the original collection of LeOpOLD II. and are 150200 years old. The connecting galleries are decorated by plenty of large and old Fuchsia plants. The greenhouses, their history, architecture and plants are described.
\end{abstract}

\section{Zusammenfassung}

Die Königlichen Gewächshäuser in Laeken wurden zwischen 1874 und 1905 gebaut. Der große Gewächshauskomplex wurde von König Leopold II., Sohn des ersten belgischen Königs Leopold I., in Auftrag gegeben. Die Königlichen Gewächshäuser sind für die Öffentlichkeit jedes Jahr von Mitte April bis Anfang Mai geöffnet. Palmenhaus, Wintergarten und die anderen beeindruckenden Gewächshäuser beherbergen eine umfangreiche Pflanzensammlung. Manche der Palmen, Kamelien und Zitrusgewächse gehen auf die ursprüngliche Sammlung von Leopold II. zurück und sind 150-200 Jahre alt. Die die Häuser verbindenden Galerien sind üppigst mit großen und alten Fuchsienpflanzen geschmückt. Die Gewächshäuser inklusive ihrer Geschichte, Architektur und Pflanzen werden vorgestellt.

\section{Einleitung}

Jedes Jahr öffnen um den 1. Mai für etwa drei Wochen die historischen Königlichen Gewächshäuser in Laeken (Brüssel). Für Freunde von Pflanzen sowie Gewächshausarchitektur ist es ein sehr beeindruckendes Erlebnis, die gläserne Stadt mit ihrer exotischen Pflanzenwelt bestaunen zu dürfen. Bezüglich seiner Größe, Architektur und des Pflanzenbestandes ist dieser Gewächshauskomplex einmalig für Europa. Dementsprechend stark frequentiert sind die Gewächshäuser, sodass es sich empfiehlt, einen Besuch gleich morgens zu beginnen, wenn es noch nicht ganz so voll ist. Die Gewächshausanlage gehört zum Schloss Laeken und ist in einen großen, nicht öffentlichen Landschaftspark eigebettet. Pflanzen und Gebäude sind der Verwaltung der Königlichen Schenkung sowie der Staatlichen Gebäudeverwaltung unterstellt.

\section{Geschichtlicher Abriss}

Das Schloss mit seinen Gärten liegt in Brüssel in fußläufiger Entfernung zum Atomium. Seine Anfänge gehen auf das 18. Jh. zurück. Es diente zunächst als Sommerresidenz von Maria Christina von Österreich (eine Tochter von Maria Theresia) und Albert von SachsenTeschen. Schon damals war es in aristokratischen Kreisen üblich, exotische Pflanzen zu halten. Die heutige Orangerie geht auf das frühe 19. Jh. zurück; sie beherbergte Zitruspflanzen, die der Landvogt KARL von Lothringen in Italien gekauft hatte. Nach der Unabhängigkeit Belgiens (1830) fiel der Besitz an den ersten belgischen König, Leopold I. (Regierungszeit: 1831 - 1865). Bereits er vergrößerte das Grundstück durch Zukauf von angrenzenden Ländereien. Entscheidende Veränderungen ließ aber erst sein Sohn Leopold II. (1835-1907, König von Belgien ab 1865) durchführen. Da er das Schloss als Begegnungs- und Kongresszentrum nutzen wollte, erweiterte er erneut das Gelände und nahm am Schloss Umbauten vor. Im Jahr 1868, also um die Gründungszeit des Palmengartens, als der Bau von großen Palmenhäusern aus technischen Gründen überhaupt erst möglich und dann sehr modern wurde, fasste der Regent den Entschluss, eine Gewächshausanlage der Superlative zu bauen. Leopold II. wollte mit der Anlage nicht nur repräsentieren, er selbst hatte nachweislich fundierte botanische Kenntnisse und war am Aufbau einer hochwertigen Pflanzensammlung persönlich sehr interessiert.

Den Auftrag der Realisierung erhielt der Architekt Alphonse Balat. In mehreren Bauphasen von 1874-1905 wurden die verschiedenen Gewächshäuser, Verbindungsgänge und Blüten- 


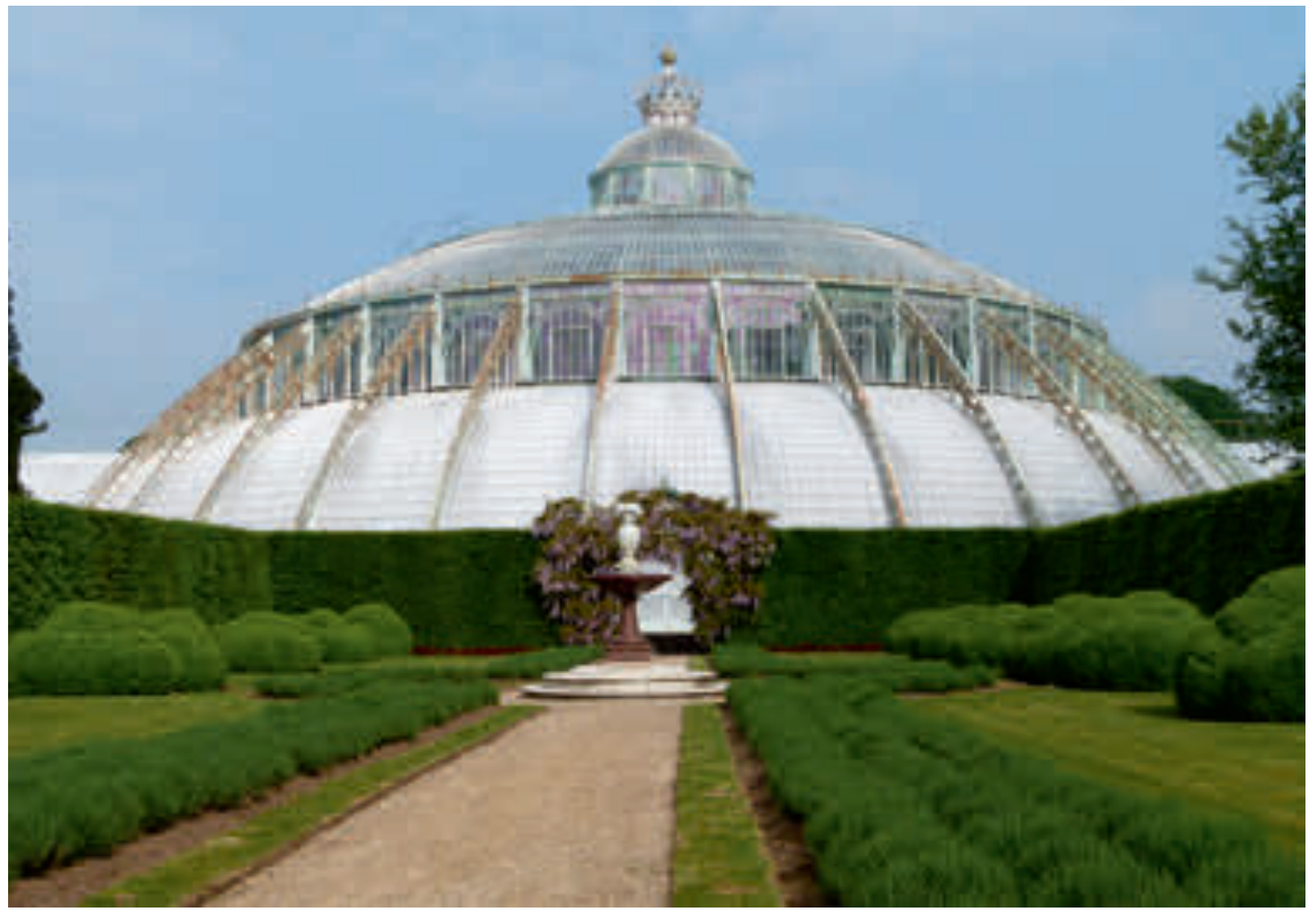

galerien gebaut. Ab 1895, nach dem Tod von Balat, waren Henri Maquet und Charles Girault für die Fertigstellung verantwortlich. Die Häuser wurden vollständig aus Eisen und Glas errichtet. Die Fläche der gesamten Domäne inkl. Schloss, Gewächshausanlage und den durch einen französischen Landschaftsarchitekten angelegten Park beträgt bis heute rund 220 ha (das ist etwa 10 Mal so viel wie die Fläche des Palmengartens!), wobei die Gewächshäuser insgesamt eine Fläche von 1,5 ha überspannen.

Um eine ganz besondere Pflanzensammlung aufzubauen, arbeitete LEOPOLD II. nur mit den besten Botanikern, Züchtern und Lieferanten zusammen. Sammlungsschwerpunkte waren Palmen, Zitrusgewächse, Kamelien und Baumfarne. Schon bald galt die Laeker Sammlung als eine der schönsten in ganz Festlandseuropa. Die ältesten der heute hier gedeihenden stattli-

Abb. 1: Der Wintergarten, das repräsentativste der Gewächshäuser, von außen betrachtet. chen Palmen dürften bereits 150 Jahre alt sein. LeOpold II. liebte seine gläserne Stadt über alles und scheute keine Kosten und Mühen, um sie im besten Zustand zu erhalten. Er selbst kümmerte sich um die Pflanzen und kontrollierte in kalten Winternächten, ob die Temperatur für die Pflanzen nicht zu niedrig sei. Von seinen verschiedenen Auslandsreisen (natürlich auch in die belgische Kolonie Kongo, deshalb auch das Kongohaus) brachte er immer wieder neue Pflanzen mit. In seinen letzten Jahren wohnte LeOpoldII. im Palmenpavillon, in dem er am 17.12.1909 starb. Den größten Anteil seines Immobilienbesitzes vererbte er dem Staat. Die verwaltende Stiftung heißt bis heute Königliche Schenkung. Für die Nachwelt hat LeOpOld II. eine großartige Gewächshausanlage hinterlassen, die bis heute so erhalten ist wie sie Leopold II. bauen ließ. Auch sein Konzept der Pflanzensammlung ist unverändert, und viele der alten Pflanzen stammen noch aus seiner Zeit.

Die Gewächshausanlage besteht aus Orangerie, Theaterhaus, Palmenhaus, Azaleenhaus, 


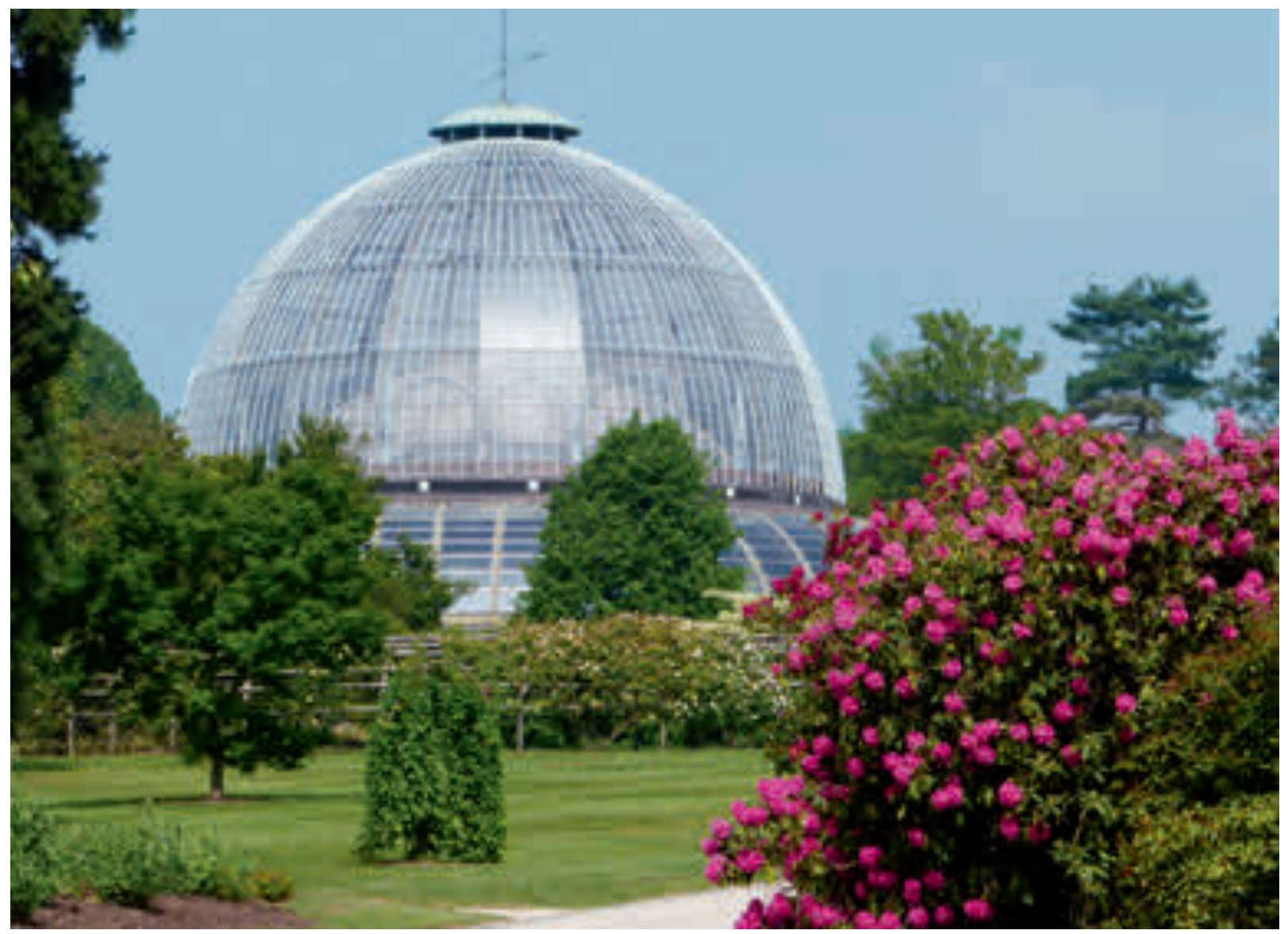

Werkstatt von Königin Elisabeth, Spiegelhaus, Embarcadère, Kongohaus und Wintergarten. Die einzelnen Häuser sind durch Galeriegänge, z.T. unterirdisch, miteinander verbunden. In ihnen gedeihen absolut prächtig, wie es üppiger nicht mehr geht, u. a. Jahrzehnte alte Hochstämme von Fuchsien und Geranien in vielen verschiedenen Sorten. Die Wände des unterirdischen Ganges sind mit Zwerg-Gummibaum (Ficus pumila) bewachsen, der ein Alter von 100 Jahren haben dürfte.

Um die Gewächshäuser und die Sammlung im guten Zustand zu erhalten, (Renovierung/ Sanierung ist ständig nötig) ist ein hoher Etat erforderlich. Um so erstaunlicher ist es, dass der Eintritt für Erwachsene nur 2 Euro beträgt. Hat man als Besucher die Schlangen an der Kasse überwunden, gelangt man durch den vorderen Teil des Parks direkt auf die $97 \mathrm{~m}$ lange und $13 \mathrm{~m}$ breite Orangerie. Beim Anblick der beiden hinter der Orangerie aufragenden Schornsteine ist schon zu erahnen, welch immensen Energiebedarf die Gewächshäuser haben. Das Rohrleitungssystem für warmes Wasser durchzieht mit einer beachtlichen Gesamtlänge von $14 \mathrm{~km}$ die Gewächshäuser. $\mathrm{Ab}$ Mitte April ist bereits ein Teil der Kübelpflanzen ins Freie geräumt. Von den Zitrusgewächsen werden hier vor allem Bitter-Orangen (Citrus aurantium) und Mandarinen (Citrus reticulata) gehalten. Aufgelockert wird das Bild durch große alte Kamelien und verschiedene Rhododendren. Ende April sind noch die weißen, extrem stark duftenden Blüten von Rhododendron fragrantissimum zu sehen. Die Kübelpflanzen sind sehr alt. Die meisten Zitruspflanzen stammen noch aus der ursprünglichen Sammlung und sind etwa 200 Jahre alt. Die

Abb. 2 (oben): Im Mai blühen im Park sehr schön alte Rhododendren.

Abb. 3 (Seite 111 oben): Das beschichtete Spezialglas erzeugt interessante Spiegeleffekte.

Abb. 4 (Seite 111 unten): Florale Verzierungen in der Gewächshauskonstruktion. 
Kollektion der Kamelien ist die älteste und bedeutendste Kameliensammlung Europas unter Glas. Während der Wochen für den öffentlichen Publikumsverkehr ist in der Orangerie ein kleiner Shop mit Gartenliteratur, Ansichtskarten und ein paar Gartenaccessoires eingerichtet. Seitlich grenzt das Theaterhaus an, das von der Königlichen Familie für Feierlichkeiten genutzt wird, im Jahr 1999 auch für die Hochzeit von Prinz Philippe und Prinzessin Mathilde.

\section{Rundgang durch die gläserne Stadt}

Von der Galerie gelangt man wieder ins Freie, um dann später von hinten durch die Gewächshäuser zurück bis zur Orangerie zu wandeln. Der Blick fällt gleich auf das zuerst fertiggestellte Gewächshaus, den Wintergarten. Er wurde 1880 eingeweiht anlässlich der Verlobung des Erzherzogs Rudolf von Österreich mit Prinzessin Stephanie. Die riesige gläserne Kuppel wird außen von 36 eisernen Rippen getragen und innen durch 36 massive Steinsäulen mit dorischen Kapitellen gestützt. Oben auf dem Dach glänzt eine vergoldete Krone. Wie auch die Scheiben der anderen Gewächshäuser irisieren sie von außen in allen Farben des Regenbogens, weil nach der erst kürzlich vorgenommenen Sanierung ein besonders beschichtetes Spezialglas (Hortiplus) eingesetzt wurde, das energiesparende Eigenschaften besitzt. Wie auch am Frankfurter Palmenhaus festgestellt wurde, war die Eisenkonstruktion ursprünglich grün gestrichen, später weiß überstrichen. Nach und nach werden nun alle Gewächshäuser aber wieder grün. Manche Glas-Partien der Gewächshäuser sind im Sommer, anstelle einer Schattierung, weiß getönt.

Auf dem Weg außen entlang der Gewächshäuser schweift der Blick über den riesigen, nicht öffentlich zugänglichen Landschaftspark mit großen Bäumen, blühenden alten Rhododendren, einem Teich und japanischer Pagode. Auffällig ist ein bizarr gedreht wachsender, alter Hänge-Schnurbaum Sophora japonica 'Pendula'. Seitlich der nicht zu besichtigenden Eisernen Kirche taucht man in die Pflanzenwelt der gläsernen Stadt ein. Hier befindet sich der Bereich des Palmenplateaus. Über einen $200 \mathrm{~m}$
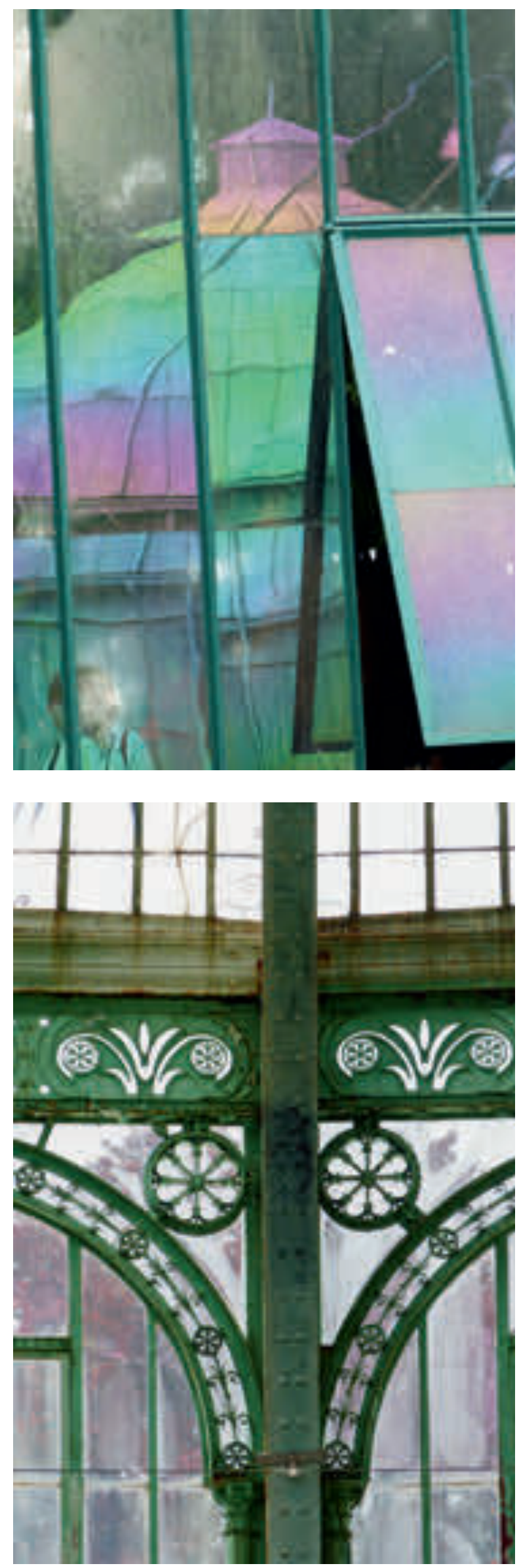

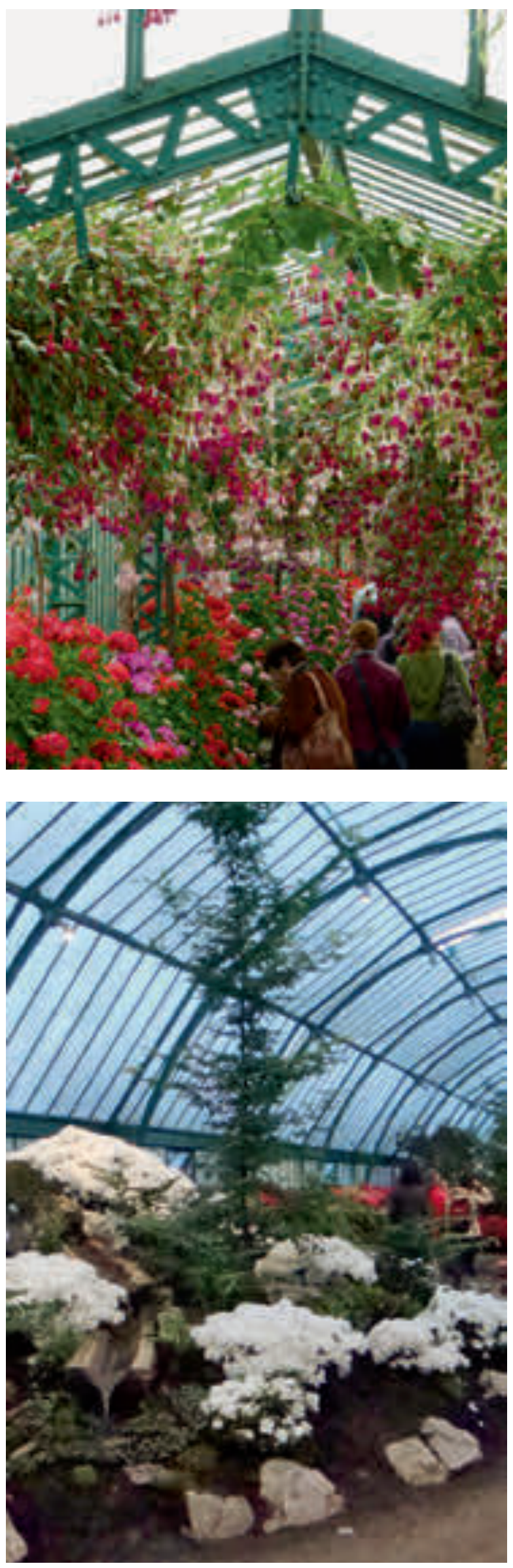

langen Gang ist er mit dem Bereich des Wintergartens verbunden. Unvorstellbar üppig sind die Gänge mit prachtvollen Pelargonien und Fuchsien ausgestattet, wobei die ältesten Fuchsien-Stämme etwa 60 Jahre alt sein sollen. Die großen Blüten hängen in Massen von den Seiten und vom Dach des Ganges herunter. Dazwischen gedeihen auch Heliotropium arborescens, Plumbago capensis und Schönmalve (Abutilon megapotamicum). Letztere zeigt die Farben Rot, Gelb und Schwarz in seinen Blüten, die die drei Farben der belgischen Nationalflagge repräsentieren sollen. Das rund $600 \mathrm{~m}^{2}$ Fläche umfassende Palmenhaus hat einen rechteckigen Grundriss, seine Eisenkonstruktion ist mit floralen Jugendstilelementen verziert. Große Exemplare der Palmen Washingtonia robusta und Chamaerops humilis, aber auch Strelitztia alba und verschiedene Gummibäume prägen das Bild. Der Boden ist von Moosfarn überwachsen und wirkt beruhigend grün.

Auf dem Weg von einem Gewächshaus ins nächste kommt man an verschiedenen dekorativ arrangierten Pflanzen vorbei. Auffällig sind u. a. auch die intensiv blau blühenden Hortensien. Das Azaleenhaus wurde 1885 errichtet. Zur Öffnungssaison sind hier Azaleen in allen Farben dekorativ arrangiert. Hier befindet sich auch eine Büste von Louis Paras, der von 1921 - 1939 Chefgärtner der königlichen Anlage war. Das Porträt wurde von Königin ElisABETH in der angegliederten Werkstatt entworfen. Aufgelockert wird das Bild durch verschiedene Kunstwerke und kunsthandwerkliche Objekte. Die Embarcadère galt als Eingangsschauhaus, wenn Gäste den Wintergarten betraten. Hier befand sich dann auch die Garderobe. In der Embarcadère stehen zwei Skulpturen von Charles van der Stappen, die

Abb. 5 (oben): Üppiger Fuchsien- und Pelargonienflor in den Verbindungsgalerien.

Abb. 6 (unten): Je nach Entwicklungszustand werden verschiedene aktuell blühende Azaleen gezeigt.

Abb. 7 (Seite 113 oben): Das Theater-Glashaus.

Abb. 8 (Seite 113 unten): Hänge-Schnurbaum (Sophora japonica 'Pendula'). 

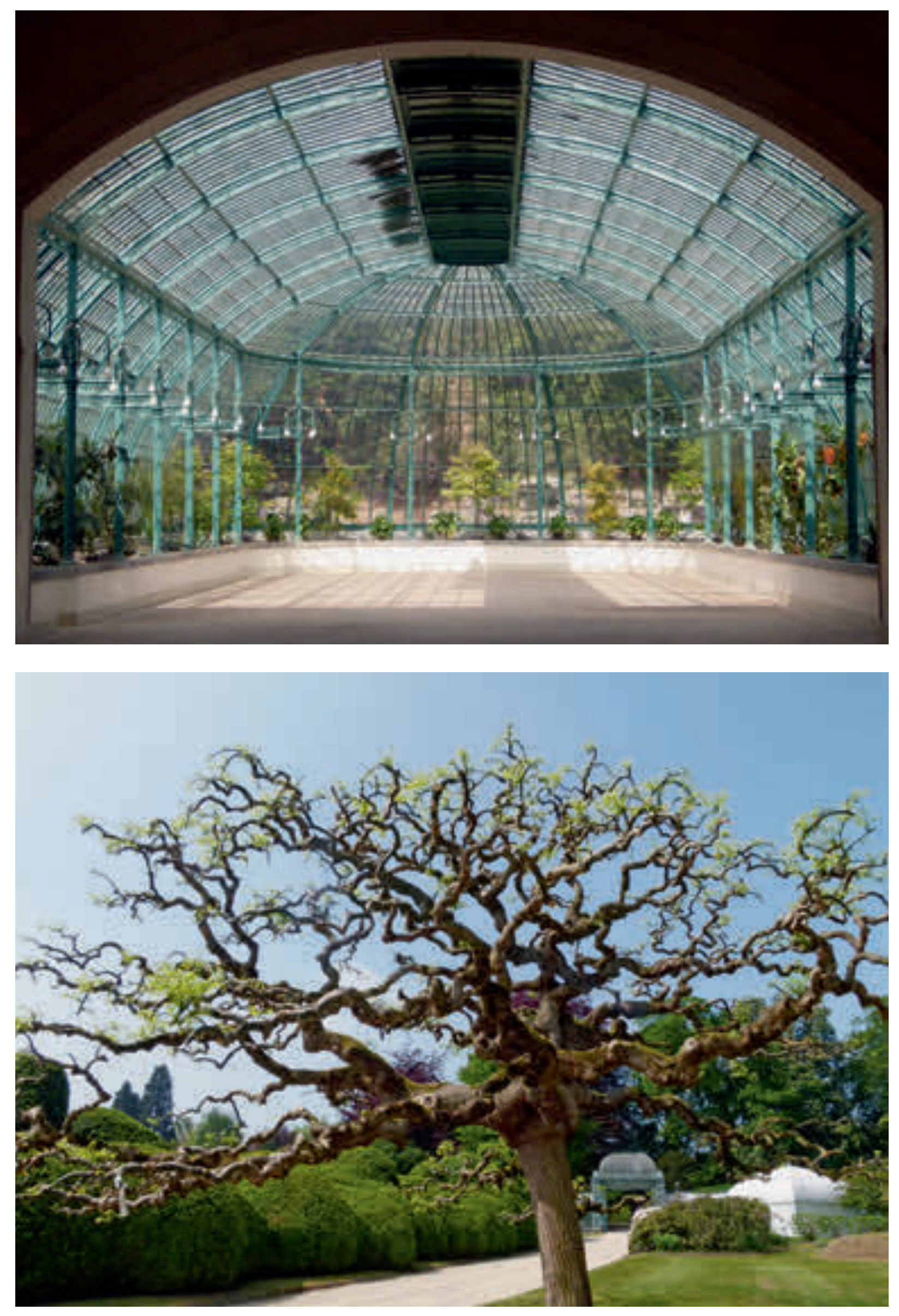

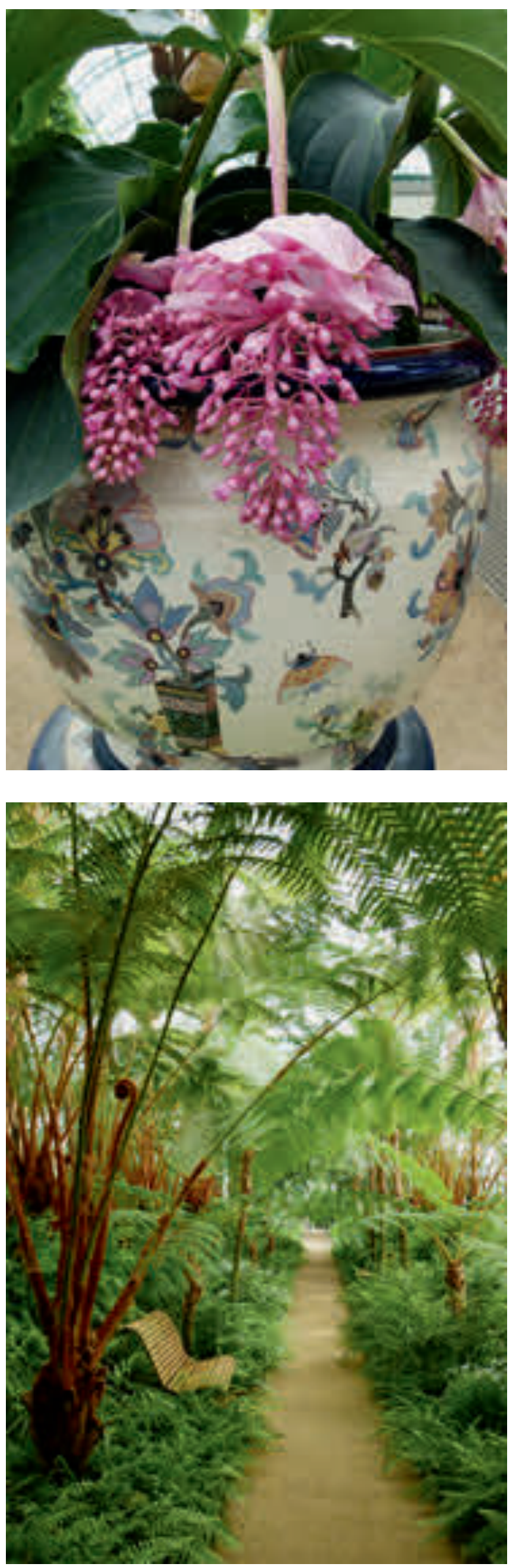

Morgenröte und der Abend. Seitlich werden Zimtbäume gezeigt. Mehrere große chinesische Vasen schaffen ein edles Ambiente. Sie sind mit Medinilla magnifica bepflanzt. Die Vasen hat LeOpold II. persönlich aus dem fernen Osten mitgebracht. Da immer die gleichen alten Medinillen in diesen Vasen gezeigt werden sollen, müssen sie immer wieder aus den Vasen entnommen und die Wurzeln zurückgeschnitten werden. Hingucker sind auch die mit Frauenhaar-Farn bepflanzten Vasen in Form von Weinbergschneckenhäusern im unterirdischen Gang. Im Dianahaus befindet sich eine Skulptur der Jagdgöttin Diana, umgeben von einer üppigen Bepflanzung, darunter auch das mit rosa Blütenbällen sehr attraktive Malvengewächs Dombeya wallichii oder je nach Saison verschiedene Orchideen.

Eines der auffälligsten großen Gewächshäuser ist das Kongohaus. Der quadratische Grundriss hat Seitenlängen von jeweils $30 \mathrm{~m}$. Das aus mehreren Kuppeln bestehende Glashaus wird von einem Stern gekrönt, dem Symbol des Kongostaates. Auch im Innern findet man die dekorativen Sterne an der Eisenkonstruktion. Ursprünglich wollte LEOPOLD II. hier Pflanzen aus dem Kongobecken zeigen. Diese gediehen aus klimatischen Gründen jedoch schlecht, sodass schließlich verschiedene andere tropische und subtropische Pflanzen verwendet wurden. Hier wachsen nun z.B. diverse Palmen, Gummibäume und Farne, der Boden ist mit Selaginella kraussiana bewachsen. Das Palmenverbindungshaus führt zum Wintergarten. Hier können die Besucher unter einer Allee aus Mexikanischem Baumfarn (Cibotium regale) spazieren. Die Blattstiele sind auffällig goldbraun beschuppt. Der Farn ist am Naturstandort sehr selten. Den Gärtnern aus Laeken ist es gelungen, diesen attraktiven Farn aus Sporen heranzuziehen und zu vermehren. Ruhebänke

Abb. 9 (oben): Medinilla in einer alten chinesischen Vase.

Abb. 10 (unten): Eine Allee aus Cybotium regale lädt zum Verweilen ein.

Abb. 11 (Seite 115): Blickachse auf Diana. 


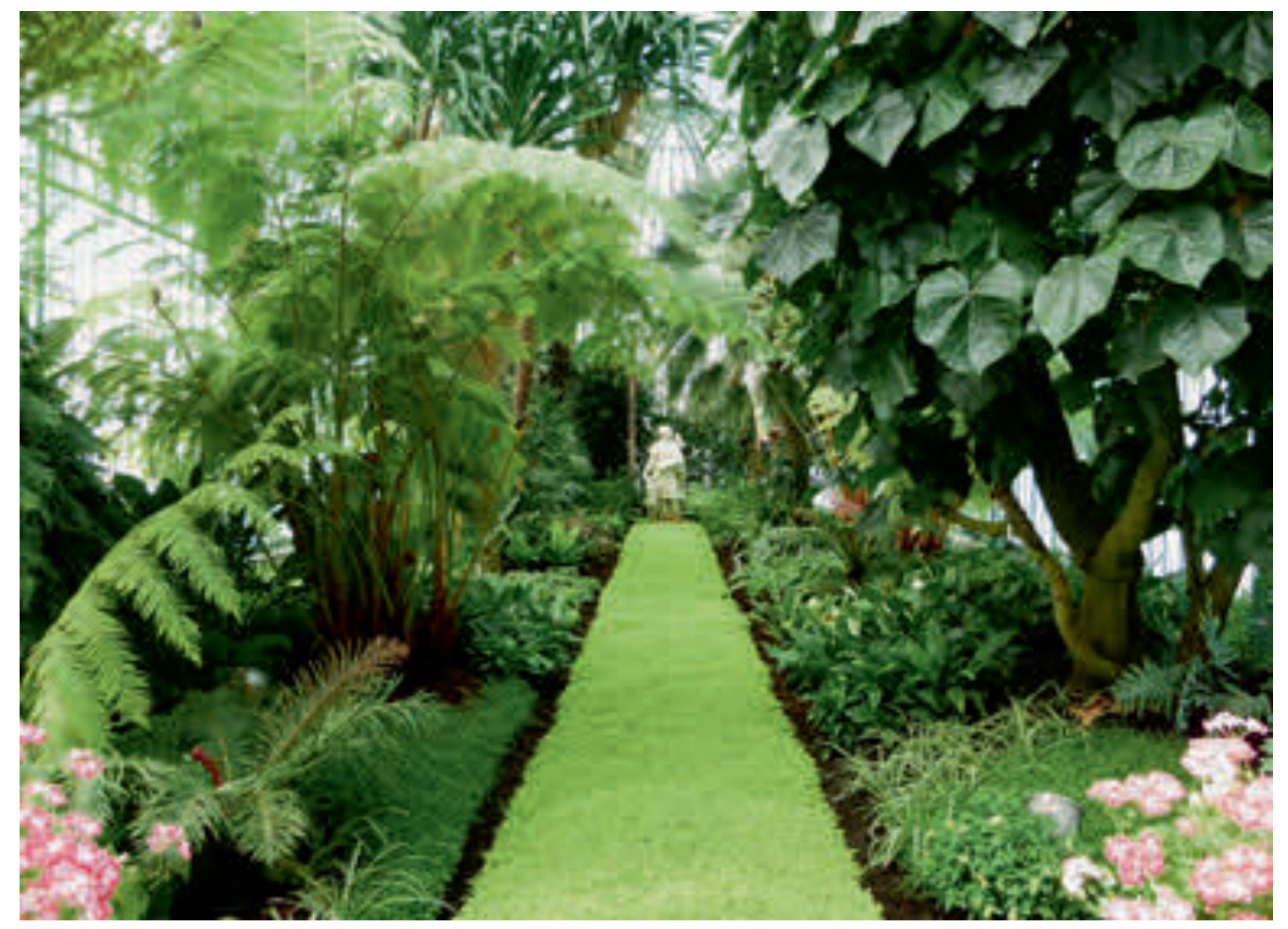

laden in diesem grün-goldenen Gewächshausareal zum Verweilen ein.

Höhepunkt schließlich ist der Wintergarten. In dem Kuppelgewächshaus mit den tragenden Säulen kommt der Eindruck eines (Pflanzen) domes auf. Leider darf man nicht ins Zentrum treten, von wo aus man den Mittelpunkt der Kuppelkonstruktion am schönsten betrachten und auf sich einwirken lassen könnte. Einige der großen Washingtonien, Kanarischen Dattelpalmen sowie Livistona chinensis und Howea forsteriana reichen fast bis unter das Dach. Der Umlauf trägt einen bunten Blütenteppich. Seitlich wachsen auch Brugmansia suaveolens und Strelitzia reginae. Die Pflanzenveteranen, vor allem die Palmen, dieses Hauses stammen noch aus der Zeit von Leopold II. oder sind zumindest direkte Nachkommen der ursprünglichen Pflanzen. Große Pflanzgefäße mit attraktiven Hortensien bereichern das Bild. Insgesamt sind die Ausmaße des Gewächshauses mit seinen großen, alten Pflanzen sehr beeindruckend: Der Wintergarten hat eine Höhe von 25,648 m, einen Innendurchmesser von 57,124 m und einen Kuppeldurchmesser von 41,254 m. Die Krone außen auf dem Dach ist $8 \mathrm{~m}$ breit. Insgesamt wurden für dieses Glashaus 651,534 Tonnen Eisen verbaut.

\section{Schlussbetrachtung}

Brüssel ist verkehrsmäßig gut angebunden und von vielen Orten aus relativ schnell erreichbar. Ein Besuch der Europa-Hauptstadt, und wenn es nur für einen Tag zum Besuch der Königlichen Gewächshäuser ist, ist jedem Pflanzenfreund nur zu empfehlen. Diese gigantische historische gläserne Gewächshausstadt mit ihrer alten, umfangreichen Pflanzensammlung schlägt alle Superlative. Da hat sich Leopold II. nicht lumpen lassen. Das Geld für diesen Luxus kam aus der Kolonie Kongo, was allerdings aus heutiger Sicht einen negativen Beigeschmack hat.

Allein die Tatsache, dass der $700 \mathrm{~m}$ lange Weg zwischen Schloss und Eiserner Kirche permanent unter Glasdächern zurückgelegt werden kann, spricht für die Einmaligkeit dieses 

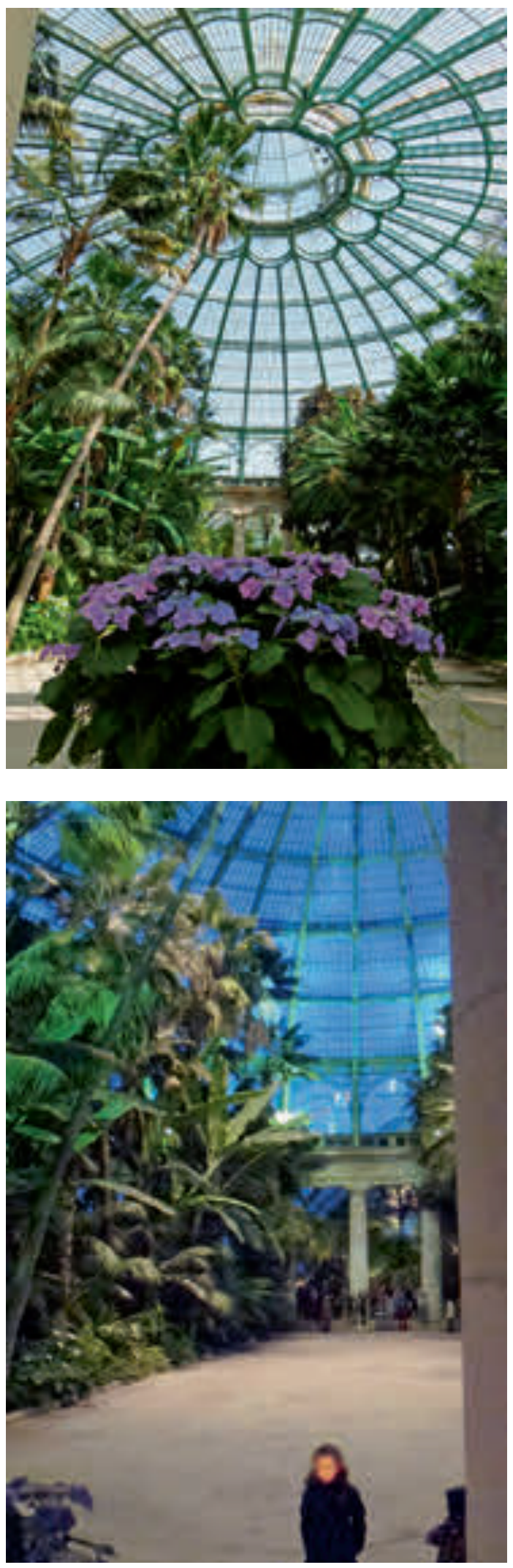

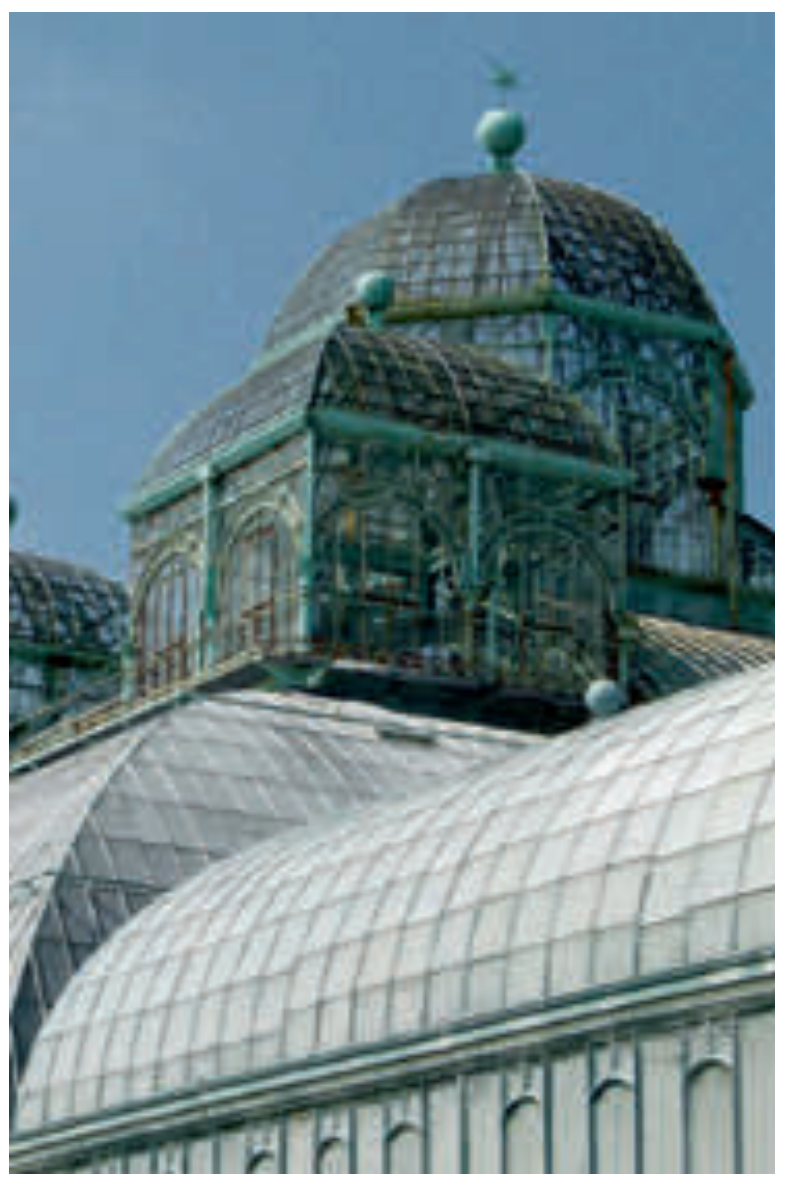

Bauprojektes. Leopold II. hatte im Alter sogar noch vorgehabt, eine unterirdische Eisenbahn bis zur Ehrentreppe der Großen Galerie des Schlosses zu bauen. Durch seinen Tod wurde dieses Vorhaben aber nicht mehr realisiert. Welch ein Glück, dass die gläserne Stadt nicht im 2. Weltkrieg zerstört wurde und uns ein eindrucksvolles Zeugnis historischer Gewächshausarchitektur mitsamt seinen wertvollen Pflanzensammlungen erhalten geblieben ist.

\section{Literatur}

SmEts, I. o. J.: Die königlichen Gewächshäuser in Laeken. - Brüssel.

Walton, G. 2014: Die königlichen Gärten in Brüssel. Gärten unter Glas. - Garten + Haus 5 (2014): 86-89.

Abb. 12 (oben links): Kongo-Stern auf der Kuppel des Kongohauses.

Abb. 13 (oben rechts): Blick von unten in die Kuppel des Wintergartens.

Abb. 14 (unten): In der Abenddämmerung wirkt der Wintergarten besonders majestätisch. 